\title{
European Option Pricing with Transaction Costs and Stochastic Volatility: an Asymptotic Analysis
}

\author{
R. E. Caflisch* G. Gambino ${ }^{\dagger}$ M. Sammartino ${ }^{\ddagger}$ C. Sgarra ${ }^{\S}$
}

June 4, 2018

\begin{abstract}
In this paper the valuation problem of a European call option in presence of both stochastic volatility and transaction costs is considered. In the limit of small transaction costs and fast mean reversion, an asymptotic expression for the option price is obtained. While the dominant term in the expansion it is shown to be the classical Black and Scholes solution, the correction terms appear at $O\left(\varepsilon^{1 / 2}\right)$ and $O(\varepsilon)$. The optimal hedging strategy is then explicitly obtained for the Scott's model.
\end{abstract}

\section{Introduction}

The intrinsic limitations of the Black-Scholes model in describing real markets behavior are very well known. Among the main assumptions underlying that model the assumptions of constant volatility and no transaction costs are probably the most relevant. In this paper we are going to consider the pricing problem of a European option in a model in which both proportional transaction costs are taken into account and the volatility is assumed to evolve according to a stochastic process of the Ornstein-Uhlenbeck type. To analyze this situation we shall follows a utility maximization procedure, following the seminal paper of M.H.A.Davis, V.G.Panas and T.Zariphopoulou [2].

If one uses the following utility function $\mathcal{U}$ :

$$
\mathcal{U}(x)=1-\exp (-\gamma x)
$$

where $\gamma$ expresses the risk aversion of the investor, one gets, as the result of this analysis, a non linear PDE for the expected value of the utility-maximized wealth held in the underlying asset of the option.

At this point we shall make two assumptions. First, that the process driving the volatility is fast mean-reverting with speed $O\left(\varepsilon^{-1}\right)$. Second, that the transaction costs are very small, $O\left(\varepsilon^{-2}\right)$.

\footnotetext{
*Department of Mathematics, UCLA, USA, rcaflisch@ipam.ucla.edu

${ }^{\dagger}$ Department of Mathematics, University of Palermo, Italy, gaetana@math.unipa.it

${ }^{\ddagger}$ Department of Mathematics, University of Palermo, Italy, marco@math.unipa.it

${ }_{\S}^{\S}$ Department of Mathematics, Politecnico di Milano, Italy, carlo.sgarra@polimi.it
} 
The pricing of a European option in presence of small transaction costs was considered in [7, where a correction term to the Black\&Scholes pricing formula was derived. This correction term was found to be order $2 / 3$ in the pricing cost. Moreover in [7] was found that the optimal hedging strategy consisted in not transacting when the process driving the stock price is in a strip around the classical Black\&Scholes delta-hedging formula and in rebalancing the portfolio (selling or buying stocks) to keep the process inside the strip of no transaction. The width of the no transaction strip was found to be order $1 / 3$ in the transaction costs. In [7] the volatility was supposed to be constant. More Recently V.I.Zakamouline studied optimal hedging of European options with transaction costs via a utility optimization approach in ([12]).

The pricing of a European option with fast mean-reverting stochastic volatility was considered in in a series of papers (see e.g. [3], [5] and [4]). In the above mentioned papers the authors found the pricing formula whose leading order term is the classical Black\&Scholes formula with averaged volatility. The correction term was order the square root of the characteristic time scale of the process driving the volatility. An optimal consumption-investment problem has been investigated in a paper by M.Bardi, A.Cesaroni and L.Manca ([8]) where a rigorous asymptotic analysis is performed and where the solution is characterized in the limit of fast volatility dynamics.

More recently, M.C.Mariani, I.SenGupta and P.Bezdek ([9]) proposed a numerical approximation scheme for European option prices in stochastic volatility models including transaction costs based on a finite-difference method. The stochastic volatility dynamics considered there is a slight generalization of that proposed by Hull and White [10], since they consider a drift coefficient which is a general (regular) deterministic function of both the time and the underlying asset price, while their diffusion coefficient is linear in the instantaneous volatility. In the present paper we propose a different approximation method for European option pricing in stochastic volatility models with transaction costs, based on an asymptotic analysis which follows the approach pioneered by J.-P.Fouque, G.Sircar and R.Papanicolaou ([3]). The model we consider for the stochastic volatility dynamics is of Ornstein-Uhlenbeck type with a constant diffusion coefficient. This model has been originally proposed by Stein and Stein [11. We provide closed-formulas for European option prices in the limit of fast volatility and small transaction costs.

The plan of the paper is the following: in Section 2 we introduce the multidimensional stochastic process for the dynamic of a portfolio of the writer of a European option. By solving a stochastic control problem, we obtain the Hamilton-Jacobi-Bellman equation. In Section 3, the asymptotic analysis is done assuming small transaction costs and fast mean reverting volatility. In section 4 the price of the option is computed and in section 5 the numerical results are provided and some conclusions outlined.

For the reader's convenience, in Appendix A the source term of the equation obtained through the asymptotic analysis at $O(\varepsilon)$ is calculated; in Appendix B the averages with respect to the Ornstein-Uhlenbeck invariant measure using the model introduced by L.Scott ([1]) for the stochastic volatility are given; finally, in Appendix $\mathrm{C}$ the derivatives, which appears in the obtained corrected pricing formula, with respect to the stock price of the classical Black and Scholes model are recalled and collected. 


\section{Option pricing via utility maximization}

We suppose to have the following multidimensional stochastic process:

$$
\begin{aligned}
d \mathbf{B} & =r \mathbf{B} d t-(1+\lambda) \mathbf{S} d \mathbf{L}+(1-\mu) \mathbf{S} d \mathbf{M} \\
d \mathbf{y} & =d \mathbf{L}-d \mathbf{M} \\
d \mathbf{S} & =\mathbf{S}(\alpha d t+f(\mathbf{z}) d \mathbf{W}) \\
d \mathbf{z} & =\xi(m-\mathbf{z}) d t+\beta\left(\rho d \mathbf{W}+\sqrt{1-\rho^{2}} d \mathbf{Z}\right) .
\end{aligned}
$$

In the above equations $\mathbf{B}$ and $\mathbf{S}$ are the risk-free (the "Bond") and the risky asset (the "Stock") respectively, $r$ is the risk-free interest rate, $\alpha$ is the drift rate of the stock, $\lambda$ and $\mu$ are the (proportional) cost of buying and selling a stock, $f$ is the volatility function, which we shall suppose to depend on the stochastic variable $z$, which is sometimes called the volatility driving process. $\mathbf{L}$ and $\mathbf{M}$ are the cumulative number of shares bought or sold, respectively, in the time interval considered $[0, T]$. We keep the notations introduced in [2] and [7], where the reader can find a detailed justification for the model for transaction costs just introduced. In what follows we shall always suppose $f(z)$ to be a function bounded away from 0 :

$$
0<m_{1} \leq f(z) \leq m_{2}<\infty, \quad \forall z
$$

The process followed by the stochastic variable $z$ is a Ornstein-Uhlenbeck process with average $m$. The parameter $\xi$ is the rate of mean reversion volatility.

The Brownian motions $\mathbf{W}$ and $\mathbf{Z}$ are uncorrelated and $\rho$ is the instantaneous correlation coefficient between asset price and the volatility shocks. Usually one considers $\rho<0$, i.e. the two processes are anti-correlated (e.g. when the prices go down the investors tend to be nervous and the volatility raises). For more details see [3] and [6].

We will suppose to deal with trading strategies absolutely continuous with respect to time, so that:

$$
\mathbf{L}=\int_{0}^{t} \mathbf{l} d s, \quad \mathbf{M}=\int_{0}^{t} \mathbf{m} d s
$$

Therefore the process we are dealing with can be written in the form:

$$
\begin{aligned}
d \mathbf{B} & =[r \mathbf{B}-(1+\lambda) \mathbf{S} \mathbf{l}+(1-\mu) \mathbf{S} \mathbf{m}] d t \\
d \mathbf{y} & =(\mathbf{l}-\mathbf{m}) d t \\
d \mathbf{S} & =\mathbf{S}(\alpha d t+f(\mathbf{z}) d \mathbf{W}) \\
d \mathbf{z} & =\xi(m-\mathbf{z}) d t+\beta\left(\rho d \mathbf{W}+\sqrt{1-\rho^{2}} d \mathbf{Z}\right) .
\end{aligned}
$$

The final value of a portfolio of the writer of a European option with strike price $K$, after following the strategy $\pi$ is:

$$
\begin{aligned}
\Phi_{w}\left(T, \mathbf{B}^{\pi}(T), \mathbf{y}^{\pi}(T), \mathbf{S}(T), \mathbf{z}(T)\right)= & \mathbf{B}^{\pi}(T)+I_{(\mathbf{S}(T)<K)} c\left(\mathbf{y}^{\pi}(T), \mathbf{S}(T)\right)+ \\
& I_{(\mathbf{S}(T)>K)}\left[c\left(\mathbf{y}^{\pi}(T)-1, \mathbf{S}(T)\right)+K\right] .
\end{aligned}
$$


On the other hand the final value of a portfolio which does not include the option is simply:

$$
\Phi_{1}\left(T, \mathbf{B}^{\pi}(T), \mathbf{y}^{\pi}(T), \mathbf{S}(T), \mathbf{z}(T)\right)=\mathbf{B}^{\pi}(T)+c\left(\mathbf{y}^{\pi}(T), \mathbf{S}(T)\right) .
$$

We can now define the following value functions:

$$
V_{j}(B)=\sup _{\pi \in \mathcal{T}} \mathbb{E}\left(\mathcal{U}\left(\Phi_{j}\left(T, \mathbf{B}^{\pi}(T), \mathbf{y}^{\pi}(T), \mathbf{S}(T), \mathbf{z}(T)\right)\right)\right)
$$

for $j=1, w$. Notice how this value functions depend on the initial endowment $B$.

Following [2] we now define:

$$
B_{j}=\inf \left\{B: V_{j}(B) \geq 0\right\} .
$$

The fair price of the option $C$ to avoid arbitrage, i.e. the amount of money that the writer has to receive to accept the obligation implicit in writing the option, will therefore be:

$$
C=B_{w}-B_{1}
$$

For this price the investor would in fact be indifferent between the two possibilities of going into the market to hedge the option, or of going into the market without the option.

We can define the following function that will be useful in the sequel:

$$
\Psi_{j}\left(T, \mathbf{B}^{\pi}(T), \mathbf{y}^{\pi}(T), \mathbf{S}(T), \mathbf{z}(T)\right)=\Phi_{j}\left(T, \mathbf{B}^{\pi}(T), \mathbf{y}^{\pi}(T), \mathbf{S}(T), \mathbf{z}(T)\right)-\mathbf{B}^{\pi}(T) .
$$

We have to find an equation for $V_{j}$. In what follows we shall suppress the index $j$ and denote $V_{j}$ with $V$. The problem we are dealing with is a stochastic control problem, where the control is the trading strategy $\mathbf{m}$ and $\mathbf{l}$. The equation for $V$ is the Hamilton-Jacobi-Bellman equation.

The Hamilton-Jacobi-Bellman equation: Suppose we have the following $n$-dimensional controlled stochastic process:

$$
d X=b(t, X, y) d t+\sigma(t, X, y) d W .
$$

Let us define the performance functional:

$$
J^{y}(s, x)=\mathbb{E}(K(T, X(T))) .
$$

In general one can define a performance functional that depends on the whole trajectory. In our case we do not need this generality.

The infinitesimal operator associated with the stochastic process is:

$$
L^{y} f=\partial_{t} f+b_{i} \partial_{x_{i}} f+a_{i j} \partial_{x_{i} x_{j}} f
$$

where

$$
a_{i j}=\frac{1}{2}\left(\sigma \sigma^{T}\right)_{i j}
$$

If one defines: 


$$
V=\sup \left\{J^{y}: y=y(x) \text { is a Markov control }\right\},
$$

then we have the following result according to the Hamilton-Jacobi-Bellman Theorem: $\sup _{y}\left\{L^{y} V\right\}=$ 0 .

In the present case this turns out to be equivalent to the following otimization problem:

$$
\begin{array}{r}
\max _{0 \leq l, m \leq k}\left\{\left(\partial_{y} V_{j}-(1+\lambda) S \partial_{B} V_{j}\right) l-\left(\partial_{y} V_{j}-(1-\mu) S \partial_{B} V_{j}\right) m+\right. \\
\partial_{t} V_{j}+r B \partial_{B} V_{j}+\alpha S \partial_{S} V_{j}+\xi(m-z) \partial_{z} V_{j}+ \\
\left.\frac{1}{2}[f(z)]^{2} S^{2} \partial_{S S} V_{j}+\frac{1}{2} \beta^{2} \partial_{z z} V_{j}+\beta f S \rho \partial_{S z} V_{j}\right\}=0 .
\end{array}
$$

With some analysis, as in [2], one gets that the above equation is equivalent to the following equation:

$$
\begin{array}{r}
\max \left\{\left(\partial_{y} V_{j}-(1+\lambda) S \partial_{B} V_{j}\right),-\left(\partial_{y} V_{j}-(1-\mu) S \partial_{B} V_{j}\right)\right. \\
\partial_{t} V_{j}+r B \partial_{B} V_{j}+\alpha S \partial_{S} V_{j}+\xi(m-z) \partial_{z} V_{j}+ \\
\left.\frac{1}{2}[f(z)]^{2} S^{2} \partial_{S S} V_{j}+\frac{1}{2} \beta^{2} \partial_{z z} V_{j}+\beta f S \rho \partial_{S z} V_{j}\right\}=0 .
\end{array}
$$

We now consider the case of the exponential utility function $\mathcal{U}(x)=1-\exp (-\gamma x)$. We note, just in passing, that this gives for $V_{j}$ the following expression:

$$
V_{j}=1-\inf \left\{\mathbb{E}\left[\exp (-\gamma B(T)) \exp \left(-\gamma \Psi_{j}\right)\right]\right\}
$$

where $\Psi_{j}$ has been previously introduced.

In the above maximization problem let us change the variables passing $V_{j} \longrightarrow W_{j}$ :

$$
V_{j}=1-\exp \left(-\frac{\gamma}{\delta}\left(B+W_{j}\right)\right)
$$

where

$$
\delta \equiv \exp [-r(T-t)] .
$$

Notice that with the above expression for $V_{j}$ the price of the option $C$, as given in (2.12), now becomes:

$$
C=W_{1}-W_{w}
$$

The maximization problem for $V_{j}$ is equivalent to the following minimization problem for $W_{j}$ :

$$
\begin{array}{r}
\min \left\{\left(\partial_{y} W_{j}-(1+\lambda) S\right),\left(-\partial_{y} W_{j}+(1-\mu) S\right)\right. \\
\partial_{t} W_{j}-r W_{j}+\alpha S \partial_{S} W_{j}+\xi(m-z) \partial_{z} W_{j}+ \\
\frac{1}{2}[f(z)]^{2} S^{2}\left[\partial_{S S} W_{j}-\frac{\gamma}{\delta}\left(\partial_{S} W_{j}\right)^{2}\right]+\frac{1}{2} \beta^{2}\left[\partial_{z z} W_{j}-\frac{\gamma}{\delta}\left(\partial_{z} W_{j}\right)^{2}\right]+ \\
\left.\beta f S \rho\left[\partial_{S z} W_{j}-\frac{\gamma}{\delta} \partial_{S} W_{j} \partial_{z} W_{j}\right]\right\}=0 .
\end{array}
$$




\section{Small transaction costs and fast mean reverting volatility: the asymptotic analysis}

We now suppose small transaction costs and fast mean reverting volatility. Moreover we will assume that the transaction costs are much smaller than the rate of mean reversion.

$$
\lambda=\mu=\varepsilon^{2}, \quad \xi=\frac{1}{\varepsilon}, \quad \beta=\frac{\sqrt{2} \nu}{\sqrt{\varepsilon}} .
$$

Buying and selling costs are assumed to be the same for simplicity.

We believe that our asymptotic assumptions are consistent with a situation where a large investor, facing very small transaction costs, is involved. In fact, in the empirical study [4] it is found that $\varepsilon \sim .005$. For large investor, typically $\lambda \lesssim .01 \%$.

In absence of transaction costs and with a deterministic volatility $\varepsilon=0$, the investor would continuously trade and get a perfect hedge staying at $y=y^{*}$, the "B\&S" hedging strategy. When transaction costs are present there is a strip of small thickness around $y=y^{*}$ where he does not transact. To resolve this strip we introduce the inner rescaled coordinate $Y$ :

$$
y=y^{*}+\varepsilon^{a} Y \quad \text { and } \quad \partial_{y} \longrightarrow \varepsilon^{-a} \partial_{Y} .
$$

The unknown boundaries between the no-transaction region and the buy and sell regions are located at:

$$
y=y^{*}+\varepsilon^{a} Y^{+} \quad \text { and } \quad y=y^{*}-\varepsilon^{a} Y^{-} .
$$

It is very important from the practical hedger point of view to determine $Y^{+}$and $Y^{-}$.

We impose the following matching conditions (see e.g. [7]):

$$
\begin{array}{ll}
W_{N T}\left(Y=Y^{ \pm}\right)=W\left(y=y^{*} \pm \varepsilon^{a} Y^{ \pm}\right) & \text {continuity } \\
\partial_{Y} W_{N T}\left(Y=Y^{ \pm}\right)=\varepsilon^{a} \partial_{y} W\left(y=y^{*} \pm \varepsilon^{a} Y^{ \pm}\right) & \text {continuity of the } \\
& \text { first derivative } \\
\partial_{Y Y} W_{N T}\left(Y=Y^{ \pm}\right)=\varepsilon^{2 a} \partial_{y y} W\left(y=y^{*} \pm \varepsilon^{a} Y^{ \pm}\right) & \text {smooth pasting } \\
\text { boundary condition }
\end{array}
$$

These boundary conditions will force, in the asymptotic analysis below, $a=1 / 3$. Therefore the strip of no-transaction will have a thickness $O\left(\varepsilon^{1 / 3}\right)$.

In the buy region $\left(Y<Y^{-}\right)$we have the equation:

$$
\left(\partial_{y} W_{j}-(1+\lambda) S\right)=0
$$

which solves to:

$$
W=(1+\lambda) S y+H^{-}(t, S, \lambda)
$$

In the sell region $\left(Y>Y^{+}\right)$we have the equation:

$$
\left(\partial_{y} W_{j}-(1-\lambda) S\right)=0 \text {, }
$$


which solves to:

$$
W=(1-\lambda) S y+H^{+}(t, S, \lambda) .
$$

In the no-transaction region we have the equation:

$$
\begin{array}{r}
\partial_{t} W_{j}-r W_{j}+\alpha S \partial_{S} W_{j}+\frac{1}{\varepsilon}(m-z) \partial_{z} W_{j} \\
+\frac{1}{2}[f(z)]^{2} S^{2}\left[\partial_{S S} W_{j}-\frac{\gamma}{\delta}\left(\partial_{S} W_{j}\right)^{2}\right]+\frac{1}{\varepsilon} \nu^{2}\left[\partial_{z z} W_{j}-\frac{\gamma}{\delta}\left(\partial_{z} W_{j}\right)^{2}\right] \\
+\frac{1}{\sqrt{\varepsilon}} \nu \sqrt{2} f S \rho\left[\partial_{S z} W_{j}-\frac{\gamma}{\delta} \partial_{S} W_{j} \partial_{z} W_{j}\right]=0
\end{array}
$$

whose solution will be find in what follows.

\subsection{The solution in the no transaction region}

As we said, in the no transaction region we use the rescaled variable $Y$ defined by (3.1). The change of variable leads to the following transformation rules for the derivatives:

$$
\begin{aligned}
\partial_{y} & \longrightarrow \varepsilon^{-1 / 3} \partial_{Y} \\
\partial_{S} & \longrightarrow \partial_{S}-\varepsilon^{-1 / 3} y_{S}^{*} \partial_{Y} \\
\partial_{t} & \longrightarrow \partial_{t}-\varepsilon^{-1 / 3} y_{t}^{*} \partial_{Y} \\
\partial_{z} & \longrightarrow \partial_{z}-\varepsilon^{-1 / 3} y_{z}^{*} \partial_{Y}
\end{aligned}
$$

We write the solution in the no transaction region in the form:

$$
W_{N T}=S\left(y^{*}+\varepsilon^{1 / 3} Y\right)+U_{0}(S, t, z)+\sum_{i=2}^{13} \varepsilon^{i / 6} U_{i}(S, t, z)+\varepsilon^{14 / 6} U_{14}(S, t, z, Y)+\ldots
$$

The derivative with respect to $t$ writes as:

$$
\partial_{t} W_{N T}=U_{0 t}+\sum_{i=2}^{11} \varepsilon^{i / 6} U_{i t}+\varepsilon^{12 / 6}\left(U_{12 t}-y_{t}^{*} U_{14 Y}\right)+\ldots
$$

The derivatives with respect to $S$ writes as:

$$
\begin{aligned}
\partial_{S} W_{N T} & =y^{*}+U_{0 S}+\varepsilon^{2 / 6}\left(Y+U_{2 S}\right)+\sum_{i=3}^{11} \varepsilon^{i / 6} U_{i S}+\varepsilon^{12 / 6}\left(U_{12 S}-y_{S}^{*} U_{14 Y}\right)+\ldots \\
\partial_{S S} W_{N T} & =U_{0 S S}+\sum_{i=2}^{9} \varepsilon^{i / 6} U_{i S S}+\varepsilon^{10 / 6}\left(U_{10 S}+\left(y_{S}^{*}\right)^{2} U_{14 Y Y}\right)+\ldots
\end{aligned}
$$

The derivatives with respect to $z$ writes as:

$$
\begin{aligned}
\partial_{z} W_{N T} & =U_{0 z}+\sum_{i=2}^{11} \varepsilon^{i / 6} U_{i z}+\varepsilon^{12 / 6}\left(U_{12 z}-y_{z}^{*} U_{14 Y}\right)+\ldots \\
\partial_{z z} W_{N T} & =U_{0 z z}+\sum_{i=2}^{9} \varepsilon^{i / 6} U_{i z z}+\varepsilon^{10 / 6}\left(U_{10 z z}+\left(y_{z}^{*}\right)^{2} U_{14 Y Y}\right)+\ldots
\end{aligned}
$$


The derivative with respect to $S$ and $z$ writes as:

$$
\partial_{S z} W_{N T}=U_{0 S z}+\sum_{i=2}^{9} \varepsilon^{i / 6} U_{i S z}+\varepsilon^{10 / 6}\left(U_{10 S z}+y_{S}^{*} y_{z}^{*} U_{14 Y Y}\right)+\ldots
$$

\subsection{The $O\left(\varepsilon^{-1}\right)$ up to $O\left(\varepsilon^{-1 / 6}\right)$ order equations}

To simplify the notation, and following the use in [5] and [6], we define the linear operators $\mathcal{L}_{i}$ and the non linear operator $\mathcal{N}_{\mathcal{L}}:$

$$
\begin{aligned}
\mathcal{L}_{0} U & =(m-z) U_{z}+\nu^{2} U_{z z} \\
\mathcal{L}_{1} U & =-\nu \sqrt{2} \rho \frac{(\alpha-r)}{f} U_{z}+\nu \sqrt{2} f S \rho U_{S z} \\
\mathcal{L}_{2} U & =U_{t}+\frac{1}{2} f^{2} S^{2} U_{S S}-r U+r S U_{S} \\
\mathcal{N}_{\mathcal{L}} U & =-\nu^{2} \frac{\gamma}{\delta}\left(U_{z}\right)^{2}
\end{aligned}
$$

The $O\left(\varepsilon^{-1}\right)$ equation is simply:

$$
\mathcal{L}_{0} U_{0}+\mathcal{N}_{\mathcal{L}} U_{0}=0
$$

The above equation can be considered an ODE in $z$ for $U_{0}$ :

$$
\nu^{2} U_{0 z z}+(m-z) U_{0 z}-\nu^{2} \frac{\gamma}{\delta}\left(U_{0 z}\right)^{2}=0 .
$$

In [6] it is proved that the only solution of an equation of this form is a $U$ which does not depend on $z$. The conclusion we therefore draw is that:

$$
U_{0} \quad \text { does not depend on } z \text {. }
$$

The $O\left(\varepsilon^{-i / 6}\right)$ equations, for $i=2, \cdots, 5$ are:

$$
\mathcal{L}_{0} U_{i}=0
$$

The conclusion is:

$$
U_{i} \quad i=2, \cdots, 5 \quad \text { does not depend on } z \text {. }
$$

\subsection{The $O(1)$ equation}

The $O(1)$ equation writes:

$$
\begin{aligned}
U_{0 t}- & r\left(S y^{*}+U_{0}\right)+\alpha S\left(y^{*}+U_{0 S}\right)+\mathcal{L}_{0} U_{6} \\
& +\frac{1}{2}[f(z)]^{2} S^{2}\left[U_{0 S S}-\frac{\gamma}{\delta}\left(y^{*}+U_{0 S}\right)^{2}\right]=0 .
\end{aligned}
$$

The above equation will be analyzed in the subsection 3.5 . 


\subsection{The $O\left(\varepsilon^{1 / 6}\right)$ equation}

The $O\left(\varepsilon^{1 / 6}\right)$ equation is:

$$
\mathcal{L}_{0} U_{7}=0 \text {. }
$$

We therefore get:

$$
U_{7} \quad \text { does not depend on } z \text {. }
$$

\subsection{The $O\left(\varepsilon^{2 / 6}\right)$ equation}

The $O\left(\varepsilon^{2 / 6}\right)$ equation is:

$$
\begin{gathered}
\mathcal{L}_{0} U_{8}+U_{2 t}-r\left(S Y+U_{2}\right)+\alpha S\left(Y+U_{2 S}\right)+ \\
+\frac{1}{2}[f(z)]^{2} S^{2}\left[U_{2 S S}-2 \frac{\gamma}{\delta}\left(y^{*}+U_{0 S}\right)\left(Y+U_{2 S}\right)\right]=0 .
\end{gathered}
$$

In the above equation there are terms that do not depend on $Y$, and terms linear in $Y$. They must be equal to zero separately. ¿From the terms linear in $Y$ one gets:

$$
y^{*}=-U_{0 S}+\frac{(\alpha-r) \delta}{f^{2} S \gamma} .
$$

The above expression gives the leading order (in absence of transaction costs) optimal hedging strategy. One recognizes the Black\&Scholes $\delta$-hedging strategy.

If one inserts the above expression into the $O(1)$ equation (3.14), one gets:

$$
\mathcal{L}_{0} U_{6}+\partial_{t} U_{0}+\frac{1}{2} f^{2} S^{2} \partial_{S S} U_{0}+r S \partial_{S} U_{0}-r U_{0}+\frac{1}{2} \frac{\delta}{\gamma} \frac{(\alpha-r)^{2}}{f^{2}}=0 .
$$

The above equation, considered as an $\mathrm{ODE}$ for $U_{6}$, is of the form:

$$
\mathcal{L}_{0} U=\chi
$$

In [5] it is shown that the solvability condition for the equation (3.18) is:

$$
\langle\chi\rangle=0,
$$

where the average $\langle\cdot\rangle$ is taken with respect to the Ornstein-Uhlenbeck process invariant measure:

$$
\langle\chi\rangle=\frac{1}{\nu \sqrt{2 \pi}} \int_{\mathbb{R}} \chi(z) e^{-(m-z)^{2} / 2 \nu^{2}} d z .
$$

Therefore the solvability condition for (3.17) is:

$$
\partial_{t} U_{0}+\frac{1}{2} \bar{\sigma}^{2} S^{2} \partial_{S S} U_{0}-r U_{0}+r S \partial_{S} U_{0}=-\frac{\delta(\alpha-r)^{2}}{2 \gamma} \frac{1}{\bar{\tau}^{2}},
$$

where $\bar{\sigma}$ is the effective constant volatility:

$$
\bar{\sigma}^{2}=\left\langle f^{2}\right\rangle
$$


and $\bar{\tau}$ is defined as:

$$
\frac{1}{\bar{\tau}^{2}}=\left\langle\frac{1}{f^{2}}\right\rangle
$$

Once one imposes to (3.21) the appropriate final condition, which will be different for the investor with option liability and the investor without it, then $U_{0}$ is determined. One can go back to equation (3.17) and solve it for $U_{6}$. The solution can be written in the form:

$$
U_{6}=U_{6}^{(z)}(S, z, t)+\tilde{U}_{6}(S, t),
$$

where, $U_{6}^{(z)}(S, z, t)$, the part of $U_{6}$ which depends on $z$, has the following expression:

$$
U_{6}^{(z)}(S, z, t)=-\mathcal{L}_{0}^{-1}\left[\frac{1}{2} S^{2}\left(f^{2}-\bar{\sigma}^{2}\right) U_{0 S S}+\frac{1}{2} \frac{\delta}{\gamma}(\alpha-r)^{2}\left(\frac{1}{f^{2}}-\frac{1}{\bar{\tau}^{2}}\right)\right] ;
$$

on the other hand $\tilde{U}_{6}$ is a function that does not depend on $z$ and that will be determined by the $O(\varepsilon)$ equation in the asymptotic procedure.

We can get a more explicit representation for $U_{6}^{(z)}$, that will be useful in the next subsection. We first define the functions $\varphi(z)$ and $\psi(z)$ as the solutions of the following problems:

$$
\begin{aligned}
\mathcal{L}_{0} \varphi & =f^{2}-\left\langle f^{2}\right\rangle, \\
\mathcal{L}_{0} \psi & =\frac{1}{f^{2}}-\left\langle\frac{1}{f^{2}}\right\rangle .
\end{aligned}
$$

Therefore the above expression for $U_{6}^{(z)}$ can be written as:

$$
U_{6}^{(z)}=-\left[\frac{1}{2} S^{2} U_{0 S S} \varphi+\frac{1}{2} \frac{\delta}{\gamma}(\alpha-r)^{2} \psi\right] .
$$

One can now go back to equation (3.15), collect all terms independent of $Y$ and get the following equation:

$$
\mathcal{L}_{0} U_{8}+\mathcal{L}_{2} U_{2}=0
$$

The above equation is a Poisson problem of the type (3.18). The solvability condition is:

$$
\left\langle\mathcal{L}_{2}\right\rangle U_{2}=0
$$

Notice that the above equation is homogeneous in $U_{2}$. Given that the final condition, both for the investor with option liability and for the investor without it, is 0 , one gets the following conclusions:

$$
\begin{aligned}
U_{2} & \equiv 0 \\
U_{8} & =\tilde{U}_{8}(S, t) \quad \text { is independent of } z .
\end{aligned}
$$

Therefore the correction to the Black and Scholes value comes up to $O\left(\varepsilon^{1 / 2}\right)$ order. 


\subsection{The $O\left(\varepsilon^{3 / 6}\right)$ equation}

Using the expression (3.16) for $y^{*}$, the $\left(\varepsilon^{3 / 6}\right)$ equation can be written as:

$$
\mathcal{L}_{2} U_{3}+\mathcal{L}_{1} U_{6}+\mathcal{L}_{0} U_{9}=0 .
$$

Notice that in the above equation appears $U_{6}$ which, until now we have derived only up to the function $\tilde{U}_{6}(S, t)$, to be determined by to a higher order asymptotics. However, in (3.27) $U_{6}$ is hit by the operator $\mathcal{L}_{1}$, which cancels $\tilde{U}_{6}(S, t)$.

Therefore one can consider (3.27) as a Poisson problem for $U_{9}$, whose solvability condition is:

$$
\left\langle\mathcal{L}_{2}\right\rangle U_{3}=-\left\langle\mathcal{L}_{1} U_{6}\right\rangle .
$$

The above equation is a Black and Scholes equation for $U_{3}$ with 0 final condition and with a source term. We now want to rewrite the source term.

By using for $U_{6}$ the expression (3.22) the operator $\mathcal{L}_{1}$ cancels the part not depending on $z$, and taking into account the expression (3.25), one can express the source term in the equation (3.28) as:

$$
\begin{array}{r}
-\left\langle\mathcal{L}_{1} U_{6}\right\rangle= \\
\left\langle\mathcal{L}_{1}\left[\frac{1}{2} S^{2} U_{0 S S} \varphi+\frac{1}{2} \frac{\delta}{\gamma}(\alpha-r)^{2} \psi\right]\right\rangle= \\
\frac{\nu \rho}{\sqrt{2}}\left[\left\langle f \varphi^{\prime}\right\rangle\left(S^{3} U_{0 S S S}+2 S^{2} U_{0 S S}\right)-(\alpha-r) S^{2} U_{0 S S}\left\langle\frac{\varphi^{\prime}}{f}\right\rangle-\frac{\delta}{\gamma}(\alpha-r)^{3}\left\langle\frac{\psi^{\prime}}{f}\right\rangle\right] .
\end{array}
$$

Therefore $U_{3}$ solves the following Black and Scholes equation:

$$
\begin{array}{r}
U_{3 t}+\frac{1}{2} \bar{\sigma}^{2} S^{2} U_{3 S S}-r U_{3}+r S U_{3 S}= \\
\frac{\nu \rho}{\sqrt{2}}\left[\left\langle f \varphi^{\prime}\right\rangle\left(S^{3} U_{0 S S S}+2 S^{2} U_{0 S S}\right)-(\alpha-r) S^{2} U_{0 S S}\left\langle\frac{\varphi^{\prime}}{f}\right\rangle-\frac{\delta}{\gamma}(\alpha-r)^{3}\left\langle\frac{\psi^{\prime}}{f}\right\rangle\right]
\end{array}
$$

with zero final data.

\subsection{The $O\left(\varepsilon^{2 / 3}\right)$ equation}

Since $U_{7}$ does not depend on $z$, the $O\left(\varepsilon^{2 / 3}\right)$ equation can be written as:

$$
\mathcal{L}_{2} U_{4}+\mathcal{L}_{0} U_{10}+\nu^{2}\left(y_{z}^{*}\right)^{2} U_{14 Y Y}-\frac{\gamma}{\delta} f^{2} S^{2} Y^{2}=0
$$

The equation (3.30) can be considered as an ODE in $Y$ for $U_{14}$. It writes as:

$$
U_{14 Y Y}=A Y^{2}+B
$$

where we have defined the following quantities: 


$$
\begin{aligned}
A & =\frac{\gamma}{\delta} \frac{f^{2} S^{2}}{\nu^{2}\left(y_{z}^{*}\right)^{2}} \\
B & =-\frac{\mathcal{L}_{2} U_{4}+\mathcal{L}_{0} U_{10}}{\nu^{2}\left(y_{z}^{*}\right)^{2}}
\end{aligned}
$$

The above equation (3.31) solves to:

$$
U_{14}=\frac{A}{12} Y^{4}+\frac{1}{2} B Y^{2}+C Y+D,
$$

with $C$ and $D$ independent of $Y$. Now we have to impose the matching conditions.

Being:

$$
\begin{array}{ll}
W_{B U Y}=\left(1+\varepsilon^{2}\right) S y+H^{-}(S, z, t) & \begin{array}{l}
\text { in the outer } \\
\text { buy region }
\end{array} \\
W_{S E L L}=\left(1-\varepsilon^{2}\right) S y+H^{+}(S, z, t) & \begin{array}{l}
\text { in the outer } \\
\text { sell region }
\end{array}
\end{array}
$$

and imposing the continuity of the gradient at the two boundaries:

$$
\begin{gathered}
\partial_{Y} W_{N T}\left(Y=-Y^{-}\right)=\varepsilon^{1 / 3} \partial_{y} W_{B U Y}\left(y=y^{*}-\varepsilon^{1 / 3} Y^{-}\right), \\
\partial_{Y} W_{N T}\left(Y=Y^{+}\right)=\varepsilon^{1 / 3} \partial_{y} W_{S E L L}\left(y=y^{*}+\varepsilon^{1 / 3} Y^{+}\right),
\end{gathered}
$$

one gets, at the $O\left(\varepsilon^{14 / 6}\right)$ :

$$
\begin{gathered}
\partial_{Y} U_{14}\left(Y=-Y^{-}\right)=S, \\
\partial_{Y} U_{14}\left(Y=Y^{+}\right)=-S .
\end{gathered}
$$

Therefore, using (3.32), one gets:

$$
\begin{aligned}
-\frac{A}{3}\left(Y^{-}\right)^{3}-B Y^{-}+C & =S, \\
\frac{A}{3}\left(Y^{+}\right)^{3}+B Y^{+}+C & =-S .
\end{aligned}
$$

Moreover, being $W$ in the outer regions linear in $y$, one imposes the continuity of the second derivative as follows:

$$
\partial_{Y Y} W_{N T}\left(Y= \pm Y^{ \pm}\right)=0,
$$

i.e.:

$$
\begin{gathered}
A\left(Y^{+}\right)^{2}+B=0 \\
A\left(Y^{-}\right)^{2}+B=0 .
\end{gathered}
$$

From these equations one sees that, at this order, the bandwidth about the Black and Scholes strategy is symmetric, i.e.:

$$
Y^{+}=Y^{-}=\left(-\frac{B}{A}\right)^{1 / 2}
$$


Subtracting the two equations (3.36) and (3.37) to eliminate $C$, and using the above expressions for $Y^{ \pm}$, one gets:

$$
\frac{2}{3}(-B)^{3 / 2} A^{-1 / 2}=S
$$

After some manipulations, and using the expressions for $A$ and $B$, the equation (3.31) leads to the following equation:

$$
\mathcal{L}_{0} U_{10}+\mathcal{L}_{2} U_{4}=\left[\frac{3}{2} f S^{2} \sqrt{\frac{\gamma}{\delta}} \nu^{2}\left(y_{z}^{*}\right)^{2}\right]^{2 / 3}
$$

One can also find an expression for the amplitude of the no-transaction region:

$$
Y^{+}=Y^{-}=\left[\frac{3}{2} \frac{1}{f^{2} S} \frac{\delta}{\gamma} \nu^{2}\left(y_{z}^{*}\right)^{2}\right]^{1 / 3} .
$$

Equation (3.39) is a Poisson problem of the type of equation (3.18). The solvability condition gives an equation for $U_{4}$ :

$$
\left\langle\mathcal{L}_{2}\right\rangle U_{4}=\left\langle\left[\frac{3}{2} f S^{2} \sqrt{\frac{\gamma}{\delta}} \nu^{2}\left(y_{z}^{*}\right)^{2}\right]^{2 / 3}\right\rangle .
$$

Notice also that, adding the two equations (3.36) and (3.37), one gets that $C=0$. Therefore:

$$
U_{14}=\frac{A}{12} Y^{4}+\frac{1}{2} B Y^{2}+D
$$

which will be useful in the subsection 3.9.

\subsection{The $O\left(\varepsilon^{5 / 6}\right)$ equation}

The $O\left(\varepsilon^{5 / 6}\right)$ equation writes as:

$$
\mathcal{L}_{0} U_{11}+\mathcal{L}_{2} U_{5}-\sqrt{2} \frac{\partial U_{6}}{\partial z} \frac{\gamma}{\delta} \nu \rho f(z) S Y-\frac{\partial U_{3}}{\partial S} \frac{\gamma}{\delta} f(z)^{2} S^{2} Y+\frac{\partial^{2} U_{15}}{\partial Y^{2}}\left(\frac{\partial y^{*}}{\partial z}\right)^{2} \nu^{2}=0
$$

This equation can be considered as an ODE for $U_{15}$ :

$$
\frac{\partial^{2} U_{15}}{\partial Y^{2}}=\bar{A} Y+\bar{B}
$$

where we have denoted:

$$
\begin{aligned}
\bar{A} & =\left(\sqrt{2} \frac{\partial U_{6}}{\partial z} \frac{\gamma}{\delta} \nu \rho f(z) S-\frac{\partial U_{3}}{\partial S} \frac{\gamma}{\delta} f(z)^{2} S^{2}\right) /\left(\nu^{2} y_{z}^{* 2}\right), \\
\bar{B} & =-\frac{\mathcal{L}_{0} U_{11}+\mathcal{L}_{2} U_{5}}{\nu^{2} y_{z}^{* 2}}
\end{aligned}
$$

Integrating (3.43) with respect to $Y$ and using the boundary conditions: 


$$
U_{15 Y}\left(Y^{+}\right)=U_{15 Y}\left(-Y^{-}\right)=0
$$

which are needed to ensure the continuity of the gradient, one gets:

$$
\mathcal{L}_{0} U_{11}+\mathcal{L}_{2} U_{5}=0 .
$$

The above equation is a Poisson problem for $U_{11}$, whose solvability condition reads:

$$
<\mathcal{L}_{2}>U_{5}=0
$$

This is a homogeneous Black-Scholes equation for $U_{5}$. Given that the final condition is zero, we gets the conclusions:

$$
\begin{aligned}
U_{5} & \equiv 0, \\
U_{11} & =\tilde{U}_{11}(S, t) \quad \text { does not depend on } z .
\end{aligned}
$$

\subsection{The $O(\varepsilon)$ equation}

Collecting the $O(\varepsilon)$ terms one gets:

$$
\begin{aligned}
\mathcal{L}_{2} U_{6}+ & \mathcal{L}_{1} U_{9}+\mathcal{L}_{0} U_{12}-\frac{1}{2} f^{2} S^{2} \frac{\gamma}{\delta}\left(U_{3 S}\right)^{2}-\nu \sqrt{2} f S \rho \frac{\gamma}{\delta} U_{3 S} U_{6 z}-y_{z}^{*}(m-z) U_{14 Y} \\
- & -\frac{\gamma}{\delta} f^{2} Y S^{2} U_{4 S}+\nu^{2}\left[-y_{z z}^{*} U_{14 Y}-2 y_{z}^{*} U_{14 Y z}+\left(y_{z}^{*}\right)^{2} U_{16 Y Y}-\frac{\gamma}{\delta}\left(U_{6 z}\right)^{2}\right]=0 .
\end{aligned}
$$

The above equation can be considered as an ODE in $Y$ for $U_{16}$ :

$$
\frac{\partial^{2} U_{16}}{\partial Y^{2}}=\tilde{A} Y+\tilde{B}
$$

where we have defined:

$$
\begin{aligned}
\tilde{A} & =\frac{\gamma}{\delta} \frac{f^{2} S^{2} U_{4 S}}{\nu^{2}\left(y_{z}^{*}\right)^{2}} \\
\tilde{B} & =-\left[\mathcal{L}_{0} U_{12}+\mathcal{L}_{1} U_{9}+\mathcal{L}_{2} U_{6}-\frac{1}{2} f^{2} S^{2} \frac{\gamma}{\delta} U_{3 S}^{2}-\nu \sqrt{2} f S \rho \frac{\gamma}{\delta} U_{3 S} U_{6 z}+\right. \\
& \left.-\nu^{2}\left(y_{z z}^{*} U_{14 Y}+2 y_{z}^{*} U_{14 Y z}+\frac{\gamma}{\delta} U_{6 z}^{2}\right)+U_{14 Y} y_{z}^{*}(m-z)\right] /\left(\nu^{2}\left(y_{z}^{*}\right)^{2}\right) .
\end{aligned}
$$

Notice that $\tilde{A}$ does not depend on $Y$ and in $\tilde{B}$ the $Y$-dependent terms appear only with their derivatives in $Y$.

We integrate the equation (3.46) from $-Y^{-}$and $Y^{+}$.

Let us use the boundary conditions:

$$
U_{16 Y}\left(Y^{+}\right)=U_{16 Y}\left(-Y^{-}\right)=0 .
$$

Moreover, being $Y^{+}=Y^{-}$and from the expression (3.42) it follows that:

$$
\int_{-Y^{-}}^{Y^{+}} U_{14 Y} d Y=0 .
$$


Integrating (3.46) we get:

$$
\mathcal{L}_{0} U_{12}+\mathcal{L}_{1} U_{9}+\mathcal{L}_{2} U_{6}=\frac{1}{2} f^{2} S^{2} \frac{\gamma}{\delta}\left(U_{3 S}\right)^{2}+\nu \sqrt{2} f S \rho \frac{\gamma}{\delta} U_{3 S} U_{6 z}+\nu^{2} \frac{\gamma}{\delta}\left(U_{6 z}\right)^{2} .
$$

The solvability condition for $U_{12}$, gives the following equation for $U_{6}$ :

$$
\left\langle\mathcal{L}_{2}\right\rangle U_{6}=-\left\langle\mathcal{L}_{1} U_{9}\right\rangle+\frac{1}{2} \bar{\sigma}^{2} S^{2} \frac{\gamma}{\delta}\left(U_{3 S}\right)^{2}+\nu \sqrt{2} S \rho \frac{\gamma}{\delta} U_{3 S}\left\langle f U_{6 z}^{(z)}\right\rangle+\nu^{2} \frac{\gamma}{\delta}\left\langle\left(U_{6 z}^{(z)}\right)^{2}\right\rangle .
$$

The main results of this section are the following:

1. Equation (3.21) for $U_{0}$;

2. Equation (3.26) for $U_{2}$ which led us to $U_{2} \equiv 0$

3. Equation (3.29) for $U_{3}$;

4. Equation (3.41) for $U_{4}$;

5. Equation (3.45) for $U_{5}$ which led us to $U_{5} \equiv 0$

6. Equation (3.47) for $U_{6}$;

7. The expression (3.16) for $y^{*}$, the center of the no-transaction region.

8. The expression (3.40) for the boundaries of the no transaction region.

\section{The price of the option}

To calculate the price of the option we now use the equation (2.16). The price will have the same asymptotic expansion as $W_{j}$ with $j=1, w$, namely:

$$
C=C_{0}+\varepsilon^{1 / 3} C_{2}+\sqrt{\varepsilon} C_{3}+\varepsilon^{2 / 3} C_{4}+\varepsilon^{5 / 6} C_{5}+\varepsilon C_{6} .
$$

Each $C_{i}$ is given by:

$$
C_{i}=U_{i}^{1}-U_{i}^{w} .
$$

To find the appropriate final conditions for the $C_{i}$, we write the final conditions for $W^{1}$ and $W^{w}$. They are, respectively:

$$
W^{1}(T)=y(T) S(T)
$$

and

$$
W^{w}(T)=y(T) S(T)-\max (S(T)-K, 0)
$$

Given the expression (3.6) one has that the final conditions for the the $U_{i}$ are the following:

$$
\begin{aligned}
& U_{i}^{1}(T)=0 \quad \text { for } \quad i=0,2,3,4,5,6 . \\
& U_{0}^{w}(T)=-\max (S(T)-K, 0) \\
& U_{i}^{w}(T)=0 \quad \text { for } \quad i=2,3,4,5,6 .
\end{aligned}
$$




\subsection{The leading order price}

To calculate the leading order price we have to calculate $U_{0}^{1}$ and $U_{0}^{w}$ where they both satisfy equation (3.21). Given the respective final conditions (4.4) and (4.5) one has that:

$$
\begin{aligned}
U_{0}^{1} & =(T-t) \frac{\delta(\alpha-r)^{2}}{2 \gamma} \frac{1}{\bar{\tau}^{2}}, \\
U_{0}^{w} & =(T-t) \frac{\delta(\alpha-r)^{2}}{2 \gamma} \frac{1}{\bar{\tau}^{2}}-C^{B S},
\end{aligned}
$$

where $C^{B S}$ is the classical pricing formula for a European call option, i.e.:

$$
C^{B S}(S, t)=S N\left(d_{1}\right)-K e^{-r(T-t)} N\left(d_{2}\right)
$$

where:

$$
d_{1}=\frac{\log (S / K)+\left(r+\frac{1}{2} \bar{\sigma}^{2}\right)(T-t)}{\bar{\sigma} \sqrt{T-t}} \quad d_{2}=d_{1}-\bar{\sigma} \sqrt{T-t},
$$

and $N(z)$ is the normal cumulative distribution function. ¿From the above expressions for $U_{0}^{j}$ one obtains:

$$
C_{0}(S, t)=C^{B S}(S, t)
$$

\subsection{The $O\left(\varepsilon^{1 / 3}\right)$ correction}

The equation for $U_{2}^{1}$ and $U_{2}^{w}$ is (3.26), a homogeneous Black and Scholes equation. In both cases the final condition is homogeneous. Therefore $U_{2}^{1} \equiv 0$ and $U_{2}^{w} \equiv 0$ and:

$$
C_{2}(S, t)=0 \text {. }
$$

\subsection{The $O\left(\varepsilon^{1 / 2}\right)$ correction}

The equation for $U_{3}^{1}$ and $U_{3}^{w}$ is equation (3.29), in both cases with homogeneous final condition. Using respectively the expressions (4.6) and (4.7) in (3.29), one has that:

$$
\begin{aligned}
U_{3}^{1}= & (T-t) \frac{\nu \rho}{\sqrt{2}} \frac{\delta}{\gamma}(\alpha-r)^{3}\left\langle\frac{\psi^{\prime}}{f}\right\rangle \\
U_{3}^{w}= & -(T-t) \frac{\nu \rho}{\sqrt{2}}\left[-\frac{\delta}{\gamma}(\alpha-r)^{3}\left\langle\frac{\psi^{\prime}}{f}\right\rangle-\left\langle f \varphi^{\prime}\right\rangle\left(S^{3} C_{3 S}^{B S}+2 S^{2} C_{S S}^{B S}\right)+\right. \\
& \left.+(\alpha-r) S^{2}\left\langle\frac{\varphi^{\prime}}{f}\right\rangle C_{S S}^{B S}\right]
\end{aligned}
$$

Therefore:

$$
C_{3}(S, t)=-(T-t) \frac{\nu \rho}{\sqrt{2}}\left[\left\langle f \varphi^{\prime}\right\rangle\left(S^{3} \partial_{S}^{3} C^{B S}+2 S^{2} \partial_{S S} C^{B S}\right)-(\alpha-r) S^{2} \partial_{S S} C^{B S}\left\langle\frac{\varphi^{\prime}}{f}\right\rangle\right](
$$

The derivatives with respect to $S$ of $C^{B S}$ are explicitly calculated in the Appendix C. Moreover, the values of $\left\langle f \varphi^{\prime}\right\rangle$ and $\left\langle\varphi^{\prime} / f\right\rangle$ are computed in Appendix B by using the Scott's model. 


\subsection{The $O\left(\varepsilon^{2 / 3}\right)$ correction}

The equation for $U_{4}^{1}$ and $U_{4}^{w}$ is equation (3.41), in both cases with homogeneous final condition. The source term for the two problems is the same: in fact $y_{z}^{*}$ has the same expression for both problems. Therefore $U_{4}^{w}=U_{4}^{1}$ and

$$
C_{4}(S, t)=0 .
$$

\subsection{The $O\left(\varepsilon^{5 / 6}\right)$ correction}

The equation for $U_{5}^{1}$ and $U_{5}^{w}$ is the homogeneous Black and Scholes equation (3.45) with zero final condition in both cases. Then:

$$
C_{5}(S, t)=0 .
$$

The correction to the price without transaction costs with stochastic volatility comes up to $O(\varepsilon)$.

\subsection{The $O(\varepsilon)$ correction}

To compute the $O(\varepsilon)$ correction we have to solve the equation (3.47). It is a Black and Scholes equation with a source term. We know that $U_{6}$ is decomposed in a part dependent on $z$ and a part that does non depend on $z$, see equation (3.22). The same decomposition holds also for $C_{6}$ :

$$
C_{6}=C_{6}^{(z)}+\tilde{C}_{6}
$$

where:

$$
C_{6}^{(z)}=U_{6}^{(z) 1}-U_{6}^{(z) w}
$$

and

$$
\tilde{C}_{6}=\tilde{U}_{6}^{(z) 1}-\tilde{U}_{6}^{w}
$$

We have already computed $U_{6}^{(z) j}$, as given in equation (3.25), we can therefore calculate $C_{6}^{(z)}$. In fact, using (3.25) and the expressions (4.6) and (4.7), one gets:

$$
\begin{aligned}
U_{6}^{(z) 1} & =-\frac{1}{2} \frac{\delta}{\gamma}(\alpha-r)^{2} \psi \\
U_{6}^{(z) w} & =\frac{1}{2} S^{2} C_{S S}^{B S} \varphi-\frac{1}{2} \frac{\delta}{\gamma}(\alpha-r)^{2} \psi
\end{aligned}
$$

which gives:

$$
C_{6}^{(z)}=-\frac{1}{2} S^{2} C_{S S}^{B S} \varphi
$$

We are now left with the task of computing $\tilde{C}_{6}$.

The equation for $C_{6}$ can be derived using equation (3.47). Subtracting the two equations relative to $U_{6}^{1}$ and $U_{6}^{w}$ one gets:

$$
\begin{aligned}
\left\langle\mathcal{L}_{2}\right\rangle C_{6} & =-\left[\left\langle\mathcal{L}_{1} U_{9}^{1}\right\rangle-\left\langle\mathcal{L}_{1} U_{9}^{w}\right\rangle\right]+\frac{1}{2} S^{2} \bar{\sigma}^{2} \frac{\gamma}{\delta}\left[\left(U_{3 S}^{1}\right)^{2}-\left(U_{3 S}^{w}\right)^{2}\right]+ \\
& +\nu \sqrt{2} S \rho \frac{\gamma}{\delta}\left[U_{3 S}^{1}\left\langle f U_{6 z}^{(z) 1}\right\rangle-U_{3 S}^{w}\left\langle f U_{6 z}^{(z) w}\right\rangle\right]+\nu^{2} \frac{\gamma}{\delta}\left[\left\langle\left(U_{6 z}^{(z) 1}\right)^{2}\right\rangle-\left\langle\left(U_{6 z}^{(z) w}\right)^{2}\right\rangle\right]
\end{aligned}
$$


This equation is a Black and Scholes equation for $C_{6}$ with source term. In the Appendix A this source term is explicitly computed and the equation (4.14) writes as:

$$
\left\langle\mathcal{L}_{2}\right\rangle C_{6}=(T-t)^{2} \hat{A}+(T-t) \hat{B}+\hat{C}
$$

where:

$$
\begin{aligned}
& \hat{A}=-\frac{\nu^{2} \rho^{2}}{4} \frac{\gamma}{\delta} S^{2} \bar{\sigma}^{2}\left[\left\langle f \varphi^{\prime}\right\rangle\left(S^{3} C_{4 S}^{B S}+5 S^{2} C_{S S S}^{B S}+4 S C_{S S}^{B S}\right)+\right. \\
& \left.-(\alpha-r)\left\langle\frac{\varphi^{\prime}}{f}\right\rangle\left(S^{2} C_{S S S}^{B S}+2 S C_{S S}^{B S}\right)\right]^{2} \\
& \hat{B}=-\nu^{2} \rho^{2} S^{2}\left(\left\langle\varphi^{\prime} f\right\rangle-\frac{1}{2}(\alpha-r)\left\langle\frac{\varphi^{\prime}}{f}\right\rangle\right)\left[\langle f \varphi ^ { \prime } \rangle \left(S^{3} C_{5 S}^{B S}+8 S^{2} C_{4 S}^{B S}\right.\right. \\
& \left.\left.+14 S C_{S S S}^{B S}+4 C_{S S}^{B S}\right)-(\alpha-r)\left\langle\frac{\varphi^{\prime}}{f}\right\rangle\left(S^{2} C_{4 S}^{B S}+4 S C_{S S S}^{B S}+2 C_{S S}^{B S}\right)\right] \\
& -\frac{\nu^{2} \rho^{2}}{2} S^{3}\left\langle f \varphi^{\prime}\right\rangle\left[\left(S^{3} C_{6 S}^{B S}+11 S^{2} C_{5 S}^{B S}+30 S C_{4 S}^{B S}+18 C_{S S S}^{B S}\right)\left\langle f \varphi^{\prime}\right\rangle\right. \\
& \left.-(\alpha-r)\left(S^{2} C_{5 S}^{B S}+6 S C_{4 S}^{B S}+6 C_{S S S}^{B S}\right)\left\langle\frac{\varphi^{\prime}}{f}\right\rangle\right] \\
& -\frac{\nu^{2} \rho^{2}}{2} \frac{\gamma}{\delta} S\left[\left\langle f \varphi^{\prime}\right\rangle\left(S^{3} C_{4 S}^{B S}+5 S^{2} C_{3 S}^{B S}+4 S C_{S S}^{B S}\right)+\right. \\
& \left.-(\alpha-r)\left\langle\frac{\varphi^{\prime}}{f}\right\rangle\left(2 S C_{S S}^{B S}+S^{2} C_{3 S}^{B S}\right)\right] \times \\
& \times\left(S^{2} C_{S S}^{B S}\left\langle\varphi^{\prime} f\right\rangle-\frac{\delta}{\gamma}(\alpha-r)^{2}\left\langle\psi^{\prime} f\right\rangle\right), \\
& \hat{C}=\nu^{2} \frac{\gamma}{\delta}\left[-\frac{1}{4} S^{4}\left(C_{S S}^{B S}\right)^{2}\left\langle\varphi^{\prime 2}\right\rangle+\frac{1}{2} \frac{\delta}{\gamma}(\alpha-r)^{2} S^{2} C_{S S}^{B S}\left\langle\varphi^{\prime} \psi^{\prime}\right\rangle\right] \\
& -\nu^{2} \rho^{2}(\alpha-r)\left[(\alpha-r) S^{2} C_{S S}^{B S}\left\langle\frac{G^{\prime}}{f}\right\rangle-\left(S^{3} C_{S S S}^{B S}+2 S^{2} C_{S S}^{B S}\right)\left\langle\frac{F^{\prime}}{f}\right\rangle\right] \\
& -\nu^{2} \rho^{2}\left[\left(S^{4} C_{4 S}^{B S}+5 S^{3} C_{S S S}^{B S}+4 S^{2} C_{S S}^{B S}\right)\left\langle F^{\prime} f\right\rangle\right. \\
& \left.-(\alpha-r)\left(S^{3} C_{S S S}^{B S}+2 S^{2} C_{S S}^{B S}\right)\left\langle G^{\prime} f\right\rangle\right] \text {. }
\end{aligned}
$$

Given the homogeneous final condition, the solution of (4.15) writes as:

$$
C_{6}=\frac{(T-t)^{3}}{3} \hat{A}+\frac{(T-t)^{2}}{2} \hat{B}+(T-t) \hat{C} .
$$

We have used the fact that:

$$
\begin{aligned}
\mathcal{L}_{2}\left(\frac{(T-t)^{3}}{3} A+\frac{(T-t)^{2}}{2} B+(T-t) C\right)= & (T-t)^{2} A+(T-t) B+C+ \\
& +\frac{(T-t)^{3}}{3} \mathcal{L}_{2} A+\frac{(T-t)^{2}}{2} \mathcal{L}_{2} B+(T-t) \mathcal{L}_{2} C
\end{aligned}
$$


and the last three terms are zero:

$$
\mathcal{L}_{2}\left(S^{n} \frac{\partial^{n} C^{B S}}{\partial S^{n}}\right)=S^{n} \frac{\partial^{n}}{\partial S^{n}} \mathcal{L}_{2} C^{B S}=0
$$

In Appendix $\mathrm{C}$ the reader can find the derivatives of $C^{B S}$ with respect to $S$ up to the sixth order. In Appendix B the averages $\langle\cdot\rangle$ with respect to the Ornstein-Uhlenbeck process invariant measure are explicitly computed using the Scott's model.

\section{$5 \quad$ Numerical results}

In this section we present the main results obtained via the asymptotic method. At first we plot the no transaction region for different values of the volatility, ranging from $5 \%$ up to $60 \%$, both in the case which does not include the option, denoted by the index 1 , and in the case which includes the option, denoted by the index $w$. The volatility is chosen as in the Scott model, $f(z)=e^{z}$. In figure 1 the curves representing the Black and Scholes strategy $y^{*}$ in the absence of transaction costs and the hedging boundaries, $y=y^{*} \pm \varepsilon^{\frac{1}{3}} Y^{+}$, are plotted versus $S$ for the first problem. From the expressions (3.16) and (3.40) it follows that these curves are respectively given by:

$$
\begin{aligned}
y^{*} & =\frac{(\alpha-r) \delta}{e^{2 z} S \gamma} \\
y & =\frac{(\alpha-r) \delta}{e^{2 z} S \gamma} \pm \varepsilon^{\frac{1}{3}}\left[\frac{6(\alpha-r)^{2} \nu^{2} \delta^{3}}{e^{6 z} S^{3} \gamma^{3}}\right]^{\frac{1}{3}} .
\end{aligned}
$$

The corresponding curves in the second case are plotted in figure 2 and their equations are:

$$
\begin{aligned}
y^{*} & =C_{S}^{B S}+\frac{(\alpha-r) \delta}{e^{2 z} S \gamma}, \\
y & =C_{S}^{B S}+\frac{(\alpha-r) \delta}{e^{2 z} S \gamma} \pm \varepsilon^{\frac{1}{3}}\left[\frac{6(\alpha-r)^{2} \nu^{2} \delta^{3}}{e^{6 z} S^{3} \gamma^{3}}\right]^{\frac{1}{3}} .
\end{aligned}
$$

Both in the figures 1 and 2 the strike price is $K=0.5$, the risk-free interest rate is $r=0.07$, the drift rate of the stock is $\alpha=0.1$, the risk aversion is $\gamma=1$, the mean volatility $\bar{\sigma}=0.2$, the time to expiry is 0.3 and $\varepsilon=1 / 200$.

Finally, in figure 3 it is shown the curve representing the classical Black and Scholes price of a European call option with the first correction obtained at $O\left(\varepsilon^{\frac{1}{2}}\right)$ and the second correction obtained at $O(\varepsilon)$. Here the parameters are chosen as $K=100, r=0.04, \alpha=0.1, \gamma=1$, $\bar{\sigma}=0.165$, the time to expiry is $3, \varepsilon=1 / 200$. In figure 3 (a) the correlation coefficient is $\rho=0$, in figure $3(\mathrm{~b})$ is $\rho=-0.2$.

We want to remark that the oscillatory behavior exhibited in figure $2 \mathrm{~b}$ ), 2c), 2d) by $y$ for small values of $S$ has been already observed by A.E. Whalley and P.Wilmott [7] also in models with constant volatility, although this feature seems to be more pronounced in the present context. Moreover the thickness of the no transaction region in presence of stochastic volatility seems to be bigger than in model with constant volatility. The $(\epsilon)^{1 / 3}$ scaling was also a relevant feature already established in [7] which is exhibited also by the present model. 


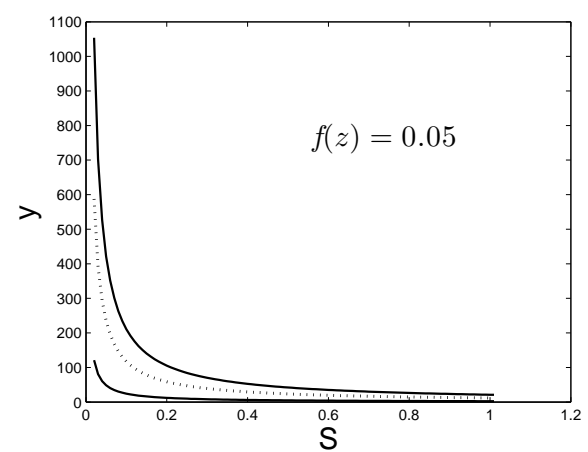

(a)

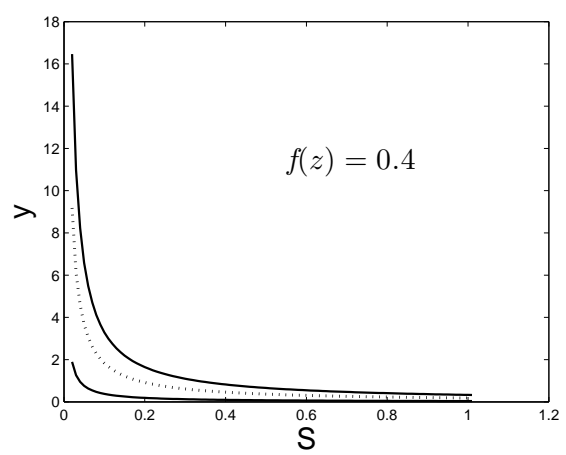

(c)

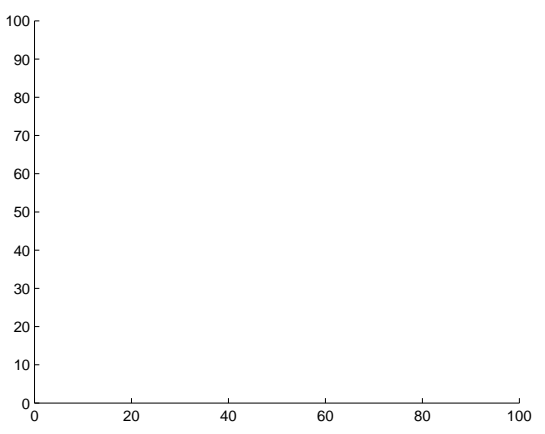

(b)

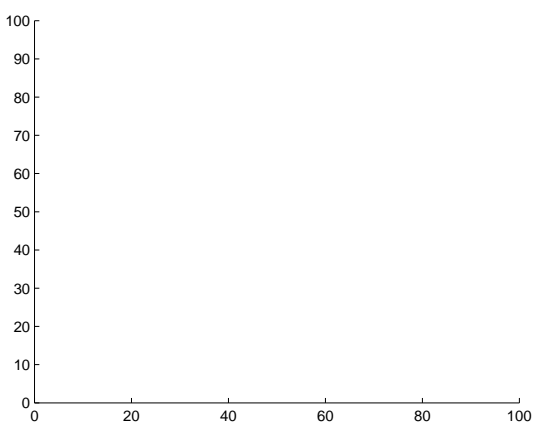

(d)

Figure 1: The no-transaction region in the case which does not include the option. The dotted curve represents the Black and Scholes strategy $y^{*}$ in the absence of transaction costs, the other two curves represent the hedging boundaries. See the text for the choice of parameters.

\section{A The source term in the equation for $C_{6}$}

\section{References}

[1] Chesney M., Scott L., Pricing European Currency Options:A Comparison of the Modified Black-Scholes Model and a Random Variance Model. J. Financial and Quantitative Analysis, 24, n.3, pp. 267-284, 1989.

[2] Davis M.A.H., Panas V.G. and Zariphopoulou T., European Option Pricing with Transaction Costs. SIAM J. Control and Optimization, 31, n.2, pp. 470-493, 1993.

[3] Fouque J.-P., Papanicolaou G., Sircar R., Derivatives in financial markets with stochastic volatility, Cambridge University Press, 2001.

[4] Fouque J.-P.,Papanicolaou G., Sircar R., Solna K., Mean reversion of S\&P 500 volatility, preprint, 1999.

[5] Fouque J.-P.,Papanicolaou G., Sircar R., Solna K., Singular Perturbation in Option Pricing. SIAM J. Appl. Math., 63, n. 5, pp. 1648-1665, 2003. 


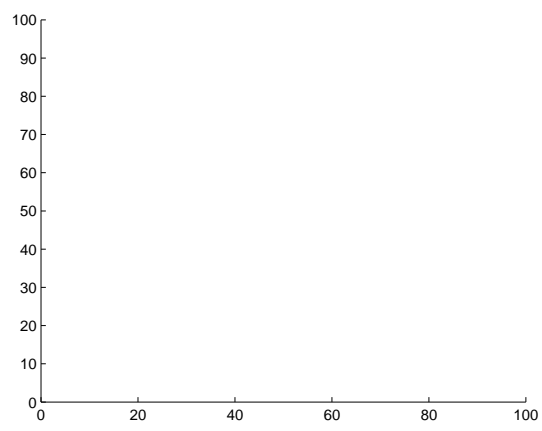

(a)

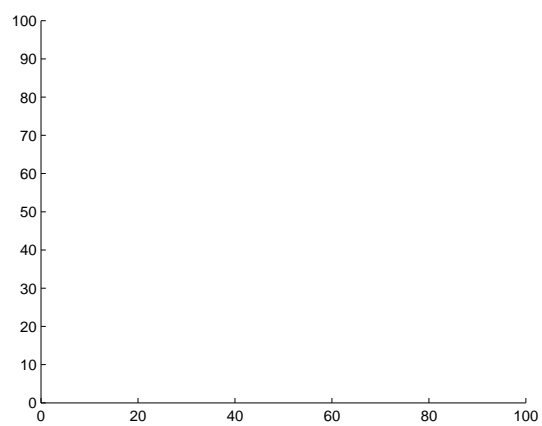

(c)

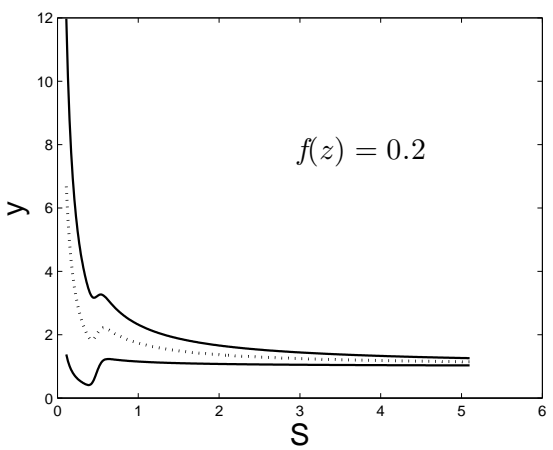

(b)

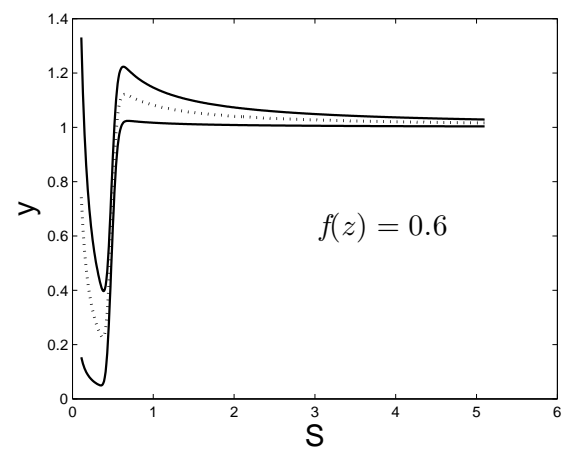

(d)

Figure 2: The no-transaction region in the case which includes the option. The dotted curve represents the Black and Scholes strategy $y^{*}$ in the absence of transaction costs, the other two curves represent the hedging boundaries. See the text for the choice of parameters.

[6] Jonsson M., Sircar R., Partial hedging in a stochastic volatility environment. Math. Finance, 12, n.4, pp. 375-4009, 2002.

[7] Whalley A. E. and Wilmott P., An Asymptotic Analysis of an Optimal Hedging Model for Option Pricing with Transaction Costs. Math. Finance, 7, n.3, pp. 307-324, 1997.

[8] Bardi M., Cesaroni A. and Manca L., Convergence by viscosity methods for a multiscale financial models with stochastic volatilities. SIAM J. Math. Finance, 1, n.1, pp. 230-265, 2010 .

[9] Mariani M.C., SenGupta I. and Bezdek P., Numerical Solutions for Option Pricing Models including Transaction Costs and Stochastic Volatility. Acta Appl. Math., 118, pp. 203-220, 2012.

[10] Hull J., White A., The Pricing of Options on Assets with Stochastic Volatility. J. Finance., 42, pp. 281-300, 1987.

[11] Stein E., Stein J., Stock Price Distributions with Stochastic Volatility: An Analytic Approach. Rev. Fin. Studies, 4, pp. 727-752, 1991. 


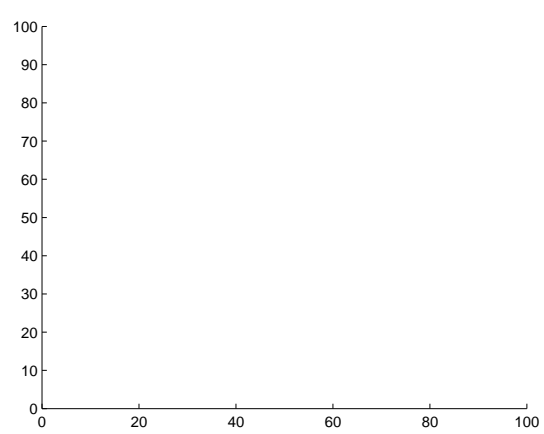

(a)

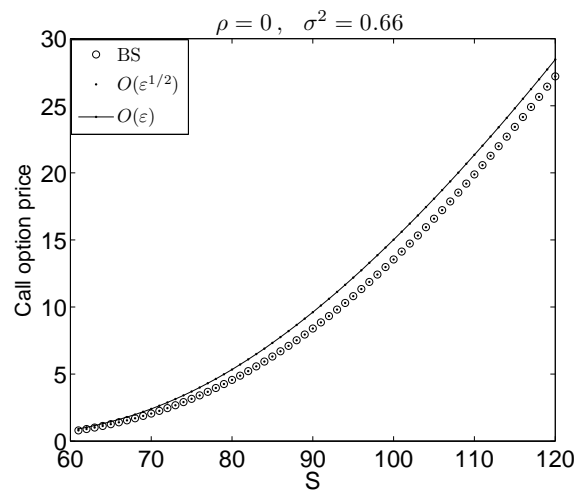

(b)

Figure 3: The dotted line represents the classical price for a European call option; the dashed line represents the price including the first correction at $O\left(\varepsilon^{\frac{1}{2}}\right)$; the continuous line represents the price including the first correction and the second correction $O(\varepsilon)$. (a) $\rho=0$; (b) $\rho=-0.2$.

[12] Zakamouline V.I., Optimal Hedging of Options with Transaction Costs. Wilmott Magazine, 118, pp. 70-82, 2006. 